\title{
CONFRONTATION OF LEMAITTRE MODELS AND THE COSMOLOGICAL CONSTANT WITH OBSERVATIONS
}

\author{
VAHÉ PETROSIAN* \\ Institute for Plasma Research, Stanford University, Stanford, Calif., U.S.A.
}

\begin{abstract}
The history of the cosmological constant and the Lemaitre models is reviewed briefly. Using recent cosmological observations, it is found that the cosmological constant if non-zero must be in absolute value less than $2 \times 10^{-56} \mathrm{~cm}^{-2}$. The predictions of the Lemaitre models are compared with modern observations. It is shown that Lemaitre models without evolution fail to reproduce the observed radio source counts. The existence of quasars with large redshift $(z>2.5)$ is shown to be strong evidence against the Lemaître models.
\end{abstract}

\section{Introduction}

Ever since its introduction by Einstein in 1917, the cosmological constant has been in and out of fashion; like an odd piece of plumbing pipe it has been found to be a useful cosmological tool on various occasions. Aside from numerous discussions pro and con the cosmological constant based primarily on philosophical arguments, there have been three occasions when it was introduced to explain some observational fact thought to be true at the time. As we shall see below, on all of these occasions subsequenct observations have changed (or have been contrary to) the original observation and sent the cosmological constant back to the shelf waiting for its next appearance.

In the next section I shall discuss the meaning and consequences of the cosmological constant and also review some of its history. The limits that can be set on the value of the cosmological constant will be discussed in Section 3. In section 4 I shall review the properties of the Lemaitre models. These models, because of their quasi-static period, are the only models whose observational characteristics are drastically different from the rest of the general relativistic models. Therefore they provide the strongest motivation for retaining the cosmological constant in the Einstein field equations. In Section 4 I shall first re-examine the motivation for the introduction of these models by Lemaitre in the 1930's, then the reason for its re-introduction by Salpeter, Szekeres and myself in 1967 and discuss them in the light of present day observations. The results and conclusions are summarized in Section 5.

\section{The Meaning of the Cosmological Constant}

\subsection{Field EQUATIONS OF GENERAL RELATIVITY}

Einstein's field equations, as proposed in 1915 are

$$
\begin{gathered}
R_{\alpha \beta}-\frac{1}{2} g_{\alpha \beta} R=\frac{8 \pi G}{c^{4}} T_{\alpha \beta}, \\
T_{\alpha \beta}=u_{\alpha} u_{\beta}(\varepsilon+p)+p g_{\alpha \beta},
\end{gathered}
$$

* Alfred P. Sloan Foundation Fellow. 
where $R_{\alpha \beta}, g_{\alpha \beta}$ and $T_{\alpha \beta}$ are the Ricci, the metric and the stress-energy tensors, respectively, $R=g^{\alpha \beta} R_{\alpha \beta}$, and $u^{\alpha}, \varepsilon$ and $p$ are the four velocity, energy density and pressure of the matter. The validity of these equations has been tested in the solar system. Whether they remain the correct description of gravitational phenomena remains to be seen. We may therefore consider them valid only for dimensions $l$ (or corresponding energy densities $\left.\varepsilon=c^{4} /\left(8 \pi G l^{2}\right)\right)$ such that

$$
l_{\min } \ll l \ll l_{\max },
$$

where $l_{\max }$ and $l_{\min }$ are much larger and smaller than a few astronomical units, respectively. One task for future investigation is to determine whether such limits exist, what their values are, and if $l_{\min } \neq 0$ and $l_{\max } \neq \infty$ how the field equations should be modified beyond these limits.

In this report I am not concerned with the lower end of the scale which must be greater than or equal to the fundamental length $l_{g}=\sqrt{G \hbar / c^{3}}=1.6 \times 10^{-33} \mathrm{~cm}$ below which quantum effects become important (cf. Ginzburg, 1971). On the high end of the scale, observations could reveal the presence of a limit if this limit is smaller than the characteristic length of the observable universe (about a few thousand $\mathrm{Mpc}$ ). One modification of the field equations which accounts for such a large scale effect was given by Einstein in 1917 when he introduced the cosmological constant $\Lambda$ :

$$
R_{\alpha \beta}-\frac{1}{2} g_{\alpha \beta} R+\Lambda g_{\alpha \beta}=\frac{8 \pi G}{c^{4}} T_{\alpha \beta} \text {. }
$$

Note that $\Lambda$ has the dimensions of inverse length squared, so that we can write $\Lambda=l_{\max }^{-2}$. The motivation for the cosmological term $\Lambda g_{\alpha \beta}$ was to obtain a static universe. But when it was discovered that the Universe is expanding, Einstein regretted the introduction of the cosmological constant and was in favor of dropping it from the field equations. Other cosmologists, however, were unwilling to abandon the more general field Equation (3). Eddington and Lemaître even claimed a logical necessity for the cosmological constant (the early history of the cosmological constant is reviewed by North (1965)). Although Einstein considered the cosmological constant 'the greatest mistake of his life' and 'detrimental to the formal beauty of his theory', Lemaitre considered it to be one of the more important contributions of Einstein.

\subsection{ENERGY DENSITY OF A VACUUM}

A major argument against the cosmological constant has been that it implies a non-zero space-time curvature even in the absence of a real stress-energy tensor (for $T_{\alpha \beta}=0$ Equation (3) reduces to $R_{\alpha \beta}=\Lambda g_{\alpha \beta}$ )*. Since, according to general relativity, curvature is produced by a stress-energy tensor, this then implies that there is a stress-energy tensor associated with a vacuum. This can be demonstrated if we define an energy density and pressure for a vacuum as

$$
\varepsilon_{v}=-p_{v}=c^{4} \Lambda / 8 \pi G \text {. }
$$

\footnotetext{
* For the Robertson-Walker metric this equation leads to the empty, expanding de Sitter universe, where test particles recede from each other at a rate proportional to $\exp \left\{\operatorname{ct}(\Lambda / 3)^{1 / 2}\right\}$.
} 
Equation (3) then reduces to Equation (1) if we replace the stress energy tensor $T_{\alpha \beta}$ by $\tilde{T}_{\alpha \beta}$ where

$$
\tilde{T}_{\alpha \beta}=T_{\alpha \beta}-\frac{c^{4} \Lambda}{8 \pi G} g_{\alpha \beta}
$$

which is identical to replacing $\varepsilon$ and $p$ in Equation (1) by

$$
\tilde{\varepsilon}=\varepsilon+\varepsilon_{v}, \quad \tilde{p}=p+p_{v} .
$$

This property of the cosmological constant was already recognized by Eddington (1939) who argued in favor of it. But many others were in favor of rejecting the $\Lambda g_{\alpha \beta}$ term because of this property.

Whether the vacuum has such a gravitational property can be settled only by observations. More recent interest in the cosmological constant has led to the speculation (cf. Zel'dovich 1968 and references cited therein) that vacuum polarization may lead to such a property. For example, as argued by Zel'dovich (1967), if a vacuum is filled with virtual pairs of particles of mass $m$ and density $n \sim(m c / \hbar)^{3}$, then the energy density due to the gravitational interaction of these pairs is $\varepsilon \sim \mathrm{Gm}^{2} /(\hbar / m c)^{4}$. This then implies that the upper limit $l_{\max }=\Lambda^{-1 / 2}$ on the range of validity of field Equation (1) is

$$
l_{\max } \sim \frac{\hbar^{2}}{G m^{3}} \sim \alpha_{G}^{-3 / 2} l_{g}
$$

where $\alpha_{G}=G m^{2} / \hbar c$ is the so called gravitational fine structure constant. For $m$ equal to the proton mass $l_{\max } \sim 1 \mathrm{Mpc}$. As we shall see such a small value for $l_{\max }$ (or the corresponding large value of $\Lambda$ ) is not compatible with observations. However, additional dimensionless parameters could be introduced in this analysis to bring the numerical value of the cosmological constant expected from vacuum fluctuations within an acceptable range.

\subsection{THE GRAVITATIONAL FORCE AND THE COSMOLOGICAL CONSTANT}

With the cosmological constant the Schwarzschild metric around a body of mass $m$ is modified to* (cf. for example Rindler, 1969)

$$
\mathrm{d} s^{2}=\left(1-\frac{2 G M}{r c^{2}}-\frac{1}{3} \Lambda r^{2}\right) \mathrm{d} t^{2}-\frac{\mathrm{d} r^{2}}{\left(1-\frac{2 G M}{r c^{2}}-\frac{1}{3} \Lambda r^{2}\right)}-r^{2}\left(\mathrm{~d} \theta^{2}+\sin ^{2} \theta \mathrm{d} \phi^{2}\right) .
$$

In the weak field limit $\left(G M / r c^{2} \ll 1\right)$ and at distances $r \ll \Lambda^{-1 / 2}$ this implies modification of the Newtonian gravitational potential and the gravitational attraction force per

* Note that the metric in Equation (8) with $m=0$ has a coordinate singularity at $r=\sqrt{3 / \Lambda}$. This is very similar to the coordinate singularity at Schwarzschild radius $r_{s}=2 G M / c^{2}$ and implies the presence of event horizon at $r=\sqrt{3 / \Lambda}$ 
unit mass:

$$
\frac{F}{m}=\frac{G M}{r^{2}}-\frac{1}{3} \Lambda c^{2} r
$$

Thus a positive (or negative) cosmological constant causes particles to repel (or attract) each other; the value of this force increases with increasing separation between particles!

Since the validity of the Schwarzschild metric without the cosmological constant has been verified in the solar system, one can set firm limits on the value of the cosmological constant. It turns out that the geodesics of zero rest-mass particles are unaffected by the $\Lambda$ term in Equation (8). But planetary orbits are modified. For example, the $\Lambda$ terms cause additional advance of the perihelion of Mercury equal to $\Lambda /(3 \times$ $\times 10^{-42} \mathrm{~cm}^{-2}$ ) seconds of arc per century (Rindler, 1969). Assuming that the observed motion of the perihelion of Mercury agrees with the $\Lambda=0$ case within $0.3^{\prime \prime}$, we obtain the limit $|\Lambda|<10^{-42} \mathrm{~cm}^{-2}$.

This, however, is not a useful limit. If $\Lambda$ were as large as this, the Newtonian equations would break down at distances of about one $\mathrm{kpc}$ from any body. This is contrary to observation of the dynamics of our Galaxy. The largest known system where Newtonian gravitational laws seem to be approximately valid are clusters of galaxies with dimensions of about one Mpc and densities of $10^{-28}$ to $10^{-29} \mathrm{~g} \mathrm{~cm}^{-3}$. For the cosmological term not to dominate* the dynamics of clusters of galaxies the value of $\varrho_{v}=\varepsilon_{v} / c^{2}$ must be less than the matter density in clusters; $\varrho_{v} \lesssim 10^{-28} \mathrm{~g} \mathrm{~cm}^{-3}$ or $|\Lambda|<$ $6 \times 10^{-55} \mathrm{~cm}^{-2}$. This limit also is larger than the values allowed from cosmological considerations, which are discussed in the next section.

\section{Limits on $\boldsymbol{A}$ from Cosmological Observations}

With the cosmological constant one obtains numerous isotropic and homogeneous cosmological models which are known as the Friedmann-Lemaitre models. These models are classified in all textbooks on cosmology. We shall follow here the classification scheme described by Petrosian and Salpeter (1968). Briefly when $\Lambda=0$ one has three kinds of models; flat, open and closed. The first two expand forever while the last possesses a high enough matter density such that its gravitational attraction is sufficiently strong to halt the expansion and cause collapse of the Universe. If $\Lambda$ is negative, the added attractive force due to it causes all three types of models to collapse into a singular state. For $\Lambda$ large and positive the repulsion due to it dominates the dynamics of the Universe so that it eventually expands like the empty de Sitter model, for which the expansion parameter goes as $a(t) \propto \exp \left\{c t(\Lambda / 3)^{1 / 2}\right\}$. This is also true for the flat and open models even for small (but positive) values of $\Lambda$. For closed world

* There is of course the problem of 'missing-mass' with clusters of galaxies. In fact a negative value of $\Lambda$ such that $\left|\varrho_{v}\right| \times($ volume of cluster $)=$ (missing-mass) could provide the additional binding force to stabilize the clusters. The required value of $\Lambda$ which provides sufficient binding is larger (in absolute value) than that allowed from cosmological considerations. 
models, the repulsion due to a positive cosmological constant can overcome the gravitational attraction of the matter in the Universe only if its value is greater than a critical value $\Lambda_{c}$. For $0<\Lambda<\Lambda_{c}$ one obtains oscillating universes. For $\Lambda=\Lambda_{c}$ one obtains either the static model of Einstein, or the model which expands from a singularity to the static models, or the Eddington-Lemaitre model which expands beginning from the Einstein static state. The Lemaitre models, which we shall discuss in the next section in more detail, have $\Lambda$ greater than but very nearly equal to $\Lambda_{c}$. For these models the expansion begins from a singularity until it reaches the Einstein state where, because of near cancellation of the gravitational attraction force of the matter in the Universe and the repulsion force due to $\Lambda$, the expansion is slowed down almost to a standstill. This is called the quasi-static period. But eventually the cosmological repulsion begins to dominate and the Universe begins to expand with an ever increasing rate. Because of this peculiar behavior, the Lemaitre model (and the limiting Eddington-Lemaitre model) have drastically different observational characters from the rest of the models.

Thus, when confronted with observation, one's first task should be to determine whether the Universe behaves like Lemaître models or like more conventional models with $\Lambda=0$ (or those with $\Lambda<0$ and those with $\Lambda>0$ but $\Lambda \neq \Lambda_{c}$ which are not very different from $\Lambda=0$ models). This will be discussed in the next section. Here we discuss the limits which the cosmological observations set on the value of the cosmological constant. We first consider the limit for negative values of the cosmological constant.

As stated above, a negative value of $\Lambda$ gives rise to models oscillating between singular points. Consequently the age of the model must exceed the age of the known constituents of the Universe. In particular, the age $t_{0}$ of the Universe must exceed the age of the Galaxy $t_{\mathrm{Gal}} \gtrsim 10^{10} \mathrm{yr}$ (for the relevant equations see Petrosian and Salpeter, 1968). Inspection of the equations shows that the age $t_{0}$ is largest for models with zero matter density $\left(\sigma_{0} \rightarrow 0\right)$ for which

$$
t_{0} H_{0} \rightarrow \frac{1}{q_{0}^{1 / 2}} \sin ^{-1}\left\{\left(\frac{q_{0}}{1+q_{0}}\right)^{1 / 2}\right\} ; \quad q_{0}=|\Lambda| c^{2} / 3 H_{0}^{2}
$$

is the deceleration parameter. Thus, the condition $t_{0}>t_{\mathrm{Gal}}$ implies that

$$
-\Lambda<3\left[\frac{\sin ^{-1} \sqrt{q_{0} /\left(1+q_{0}\right)}}{c t_{\mathrm{Gal}}}\right]^{2}
$$

with the firm limit $-\Lambda<3 \pi^{2} /\left(2 t_{\mathrm{Gal}} c\right)^{2}$. However, the observed redshift-magnitude relation of the brightest galaxies in clusters indicates (Sandage, 1972a) that the deceleration parameter $q_{0}$ is near unity. Assuming a generous upper limit of $q_{0}<3$ Equation (10) gives

$$
-\Lambda<3 \times 10^{-56} \mathrm{~cm}^{-2}\left(t_{\mathrm{Gal}} / 10^{10} \mathrm{yr}\right)^{-2} .
$$

For positive values of $\Lambda$ the limits from age considerations are not very useful since the Lemaitre models can have a large value of $\Lambda$ without violating the limit 
set on the age of the Universe from galactic evolution. In order to set limits on positive values of $\Lambda$ let us consider the equation

$$
\frac{2 \varrho_{v}}{\varrho_{c}}=\frac{\Lambda c^{2}}{3 H_{0}^{2}}=\lambda a_{0}^{3} \sigma_{0}=\frac{\lambda a_{0}^{3}}{2-3 k a_{0}+\lambda a_{0}^{3}} .
$$

Here $a_{0}$ and $\sigma_{0}$ are dimensionless expansion and density parameters, $\lambda=\Lambda / \Lambda_{c}$, and $\varrho_{c}=3 H_{0}^{2} / 4 \pi G$.

For open and flat $(k=-1,0)$ models the maximum of this quantity is unity;

$$
\Lambda<3\left(H_{0} / c\right)^{2}=0.9 \times 10^{-56} \mathrm{~cm}^{-2}\left(H_{0} / 50 \mathrm{~km} \mathrm{~s}^{-1} \mathrm{Mpc}^{-1}\right)^{2} .
$$

But for closed $(k=+1)$ models the maximum of the quantity on the right hand side of Equation (12) occurs for $a_{0}=1\left(\sigma_{0}^{-1}=\varepsilon=\lambda-1, \lambda=\Lambda / \Lambda_{c}\right)$ and is

$$
\frac{\Lambda c^{2}}{3 H_{0}^{2}}<\frac{\lambda}{\varepsilon}=\lambda \sigma_{0} \text {. }
$$

Thus large values of $\Lambda$ are possible only for Lemaitre models $(\varepsilon \ll 1)$ with the present value of the expansion parameter $a_{0}$ near unity. This, however, means that we are living not far from the quasi-static period and that the matter density $\varrho_{0}=\varrho_{c} / \varepsilon$ is very large. Furthermore, this also implies that the redshift $z_{s}=a_{0}-1$ of the quasi-static period is very small. In Lemaître models one expects many bright sources with redshifts near $z_{s}$ (cf. Section 4.4) and very few sources with larger redshifts. Therefore, a small value of $z_{s}$ is contrary to observation of galaxies and quasars (if the redshifts of these objects are cosmological in origin). If we assume $z_{s} \geqslant 1$ (which implies $a_{0} \geqslant 2$, $\left.\sigma_{0} \geqslant 0.25\right)$ we then obtain

$$
\begin{aligned}
& \Lambda<6\left(H_{0} / c\right)^{2}=2 \times 10^{-56} \mathrm{~cm}^{-2} \times\left(H_{0} / 50 \mathrm{~km} \mathrm{~s}^{-1} \mathrm{Mpc}^{-1}\right)^{2}, \\
& \varrho_{v}<\varrho_{c}=0.94 \times 10^{-29} \mathrm{~g} \mathrm{~cm}^{-3} \times\left(H_{0} / 50 \mathrm{~km} \mathrm{~s}^{-1} \mathrm{Mpc}^{-1}\right)^{2} .
\end{aligned}
$$

Equations (11) and (14) give the range of possible values for the cosmological constant. It is clear that the cosmological constant, if non-zero, will play a minor role in the dynamics of regions of the Universe smaller than $2500 \mathrm{Mpc}$ or regions with matter densities larger than $10^{-29} \mathrm{~g} \mathrm{~cm}^{-3}$. In spite of these limitations, there are many models with non-zero values of the cosmological constant which agree with known observations. In the next section we compare the Lemaitre models (which require the largest value of the cosmological constant and have quite different observational properties from the conventional models) with observations.

\section{Comparison of the Lemaître Models with Observations}

In this section we review the properties of Lemaitre models with long quasi-static periods and compare them with observation. These models are discussed in detail by Petrosian and Salpeter (1968). We shall follow the parametrization of these models as given in that article. We (Petrosian and Salpeter, 1970) have also reviewed briefly 
some observational consequences of these models. I shall re-examine here these aspects of the models in light of more recent observations. We shall concentrate on four observational aspects of these models:

(A) Age of the models and formation of galaxies.

(B) Magnitude-redshift relation.

(C) Radio source counts.

(D) Observations related to quasars.

To summarize the properties of these models, I have plotted on Figure 1 the variation of the expansion parameter $a(t)$ (in units of its present value $a_{0}=a\left(t_{0}\right)$, where $t_{0}$ is the present age of the Universe) vs the age. As shown by the upper and right hand side coordinates, this could also be considered as a plot of the co-moving coordinate $u$ of sources vs their redshifts $z$. The value of the redshift corresponding to the quasi-static period is $z_{s}=a_{0}-1$. For models with long quasistatic periods an observer can see sources at his antipode $u=\pi$, at his own position $u=2 \pi$, at its antipode again, $u=3 \pi$, etc. This allows an observer to see his own image. For example, if the Andromeda galaxy existed at the time corresponding to $u=2 \pi$ we should see another image, a ghost image, of it in a direction diametrically opposite its actual position in the sky. This, however, is unlikely because the light travel time from $u=2 \pi$ to $u=0$ (a few Hubble times) is longer than the age of ordinary galaxies. When the Lemaitre models were proposed to explain the preponderance of quasars near redshift 2 (Petrosian et al., 1967), an excess of pairs of radio sources in diametrically opposite directions was searched for. There have been claims (Solheim, 1968) that

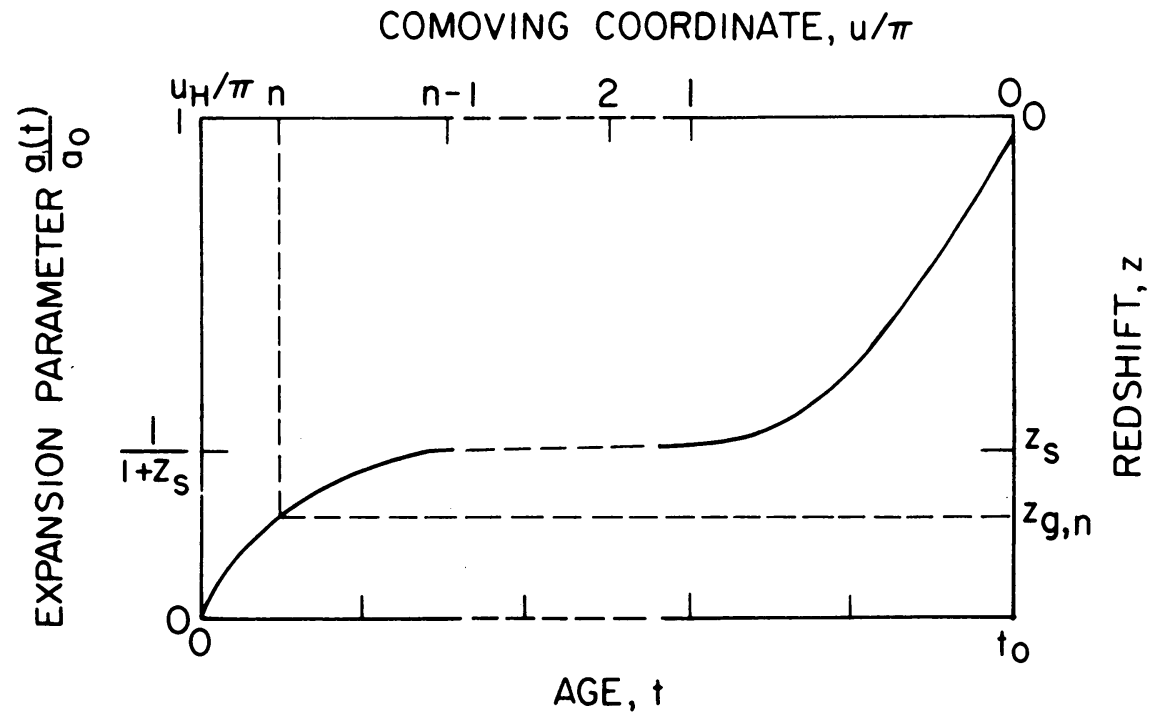

Fig. 1. Variation of expansion parameter or the redshift with time or the co-moving coordinate $u$ in a Lemaitre model. $u / \pi=i, i=1,2, \ldots, n$ are the antipodes of the model. The length of the quasistatic period (dashed line) can be made arbitrarily large by choosing a cosmological constant arbitrarily near the critical value $\Lambda_{c}$. Note for all ghost images $(u=i \pi)$, except the last one $(i=n)$, the redshift $z_{g . i} \simeq z_{s}$. 
such image pairs have been observed. But a larger sample of radio sources were examined for this effect with negative results (Petrosian and Ekers, 1969). These results, however, are still consistent with the Lemaitre models because (i) this effect should be masked by the motion and finite lifetime of sources, and (ii) inhomogeneities in the intervening medium (galaxies and cluster of galaxies) deflect the ghost images and split them into many weaker images (for details cf., Petrosian and Salpeter, 1968).

The general relations between the co-moving coordinate, the redshifts of ghost images (sources at $u=n \pi, n=1,2, \ldots$ ) and the age of the models are given in our paper for general Lemaître models. We shall be concerned primarily with models for which the redshift of the quasi-static period is $z_{s}=2$ (i.e., models with $\sigma_{0}=0.05, a_{0}=3$ ) for which we obtain

$$
\begin{aligned}
& u_{H}=4.13-\ln \varepsilon, \\
& t_{0} H_{0}=1.57-0.5 \ln \varepsilon,
\end{aligned}
$$

where $u_{H}=u(z=\infty)$ is the co-moving coordinate of the horizon. Furthermore, we find that for this model the redshifts of the ghost images, if not too different from $z_{s}$, are

$$
\begin{aligned}
& z_{g, 1}=1.89+7.2 \varepsilon \\
& \frac{z_{g, 2}=1.99+155 \varepsilon}{z_{g, n}=2.00+0.29 \varepsilon} e^{n \pi} .
\end{aligned}
$$

Note that as long as $\varepsilon e^{i \pi} \ll 1, z_{g, i} \approx z_{s}$, but since $e^{\pi} \approx 23 \gg 1$ only one ghost image (the last one) could have a redshift substantially different from $z_{s}$.

\subsection{Age AND fOrmation Of GalaXies}

As can be seen from Equation (15) the age of the Lemaitre models can be made arbitrarily large by choosing an arbitrarily small value for $\varepsilon$; the smaller the value of $\varepsilon$ the larger the duration of the quasi-static period. Prior to recalibration of the periodluminosity relation of Cepheid variables, the value of the Hubble constant was thought to be about $500 \mathrm{~km} \mathrm{~s}^{-1} \mathrm{Mpc}^{-1}$, which meant an age of less than two billion years for all Friedmann-Lemaitre models except for the models with long quasi-static periods. This was a strong motivation for considering the latter models seriously. These models have come to be known as Lemaitre models since he was the strongest advocate of these models. Since then, however, the value of the Hubble constant has been steadily decreasing. The modern value of $H_{0} \approx 50 \mathrm{~km} \mathrm{~s}^{-1} \mathrm{Mpc}^{-1}$ (Sandage, $1972 \mathrm{~b}$ ) increases the age of the conventional models to $20 \mathrm{~b}$. y., in agreement with the age of the oldest stars in our Galaxy (Iben and Faulkner, 1968).

Another reason for considering the Lemaitre models seriously had to do with the formation of galaxies. In an expanding universe the rate of growth of condensation with time is proportional to some power of time. This is not rapid enough to allow formation of condensations from statistical fluctuations. In Lemaitre models the condensations could grow exponentially during the quasi-static period (cf. for ex- 
ample Bonnor, 1957; Brecher and Silk, 1969). However, in order for condensations to form from statistical fluctuations, the quasi-static period must have exceedingly long duration, $\varepsilon<10^{-15}$.

Models with such a long quasi-static period, like the Einstein static model, may be unstable to the formation of condensations. The stability of the static models was examined by various authors during the period 1930 to 1950 which is reviewed by North (1965). Most of the investigations during this period were concerned with stability against the formation of condensations without considering the most important effect, namely the changes in the equation of state during the formation of these condensations. Lemaitre (1931), however, did consider this effect. He argued that during the formation of condensations from a gas with temperature $T$, the pressure is reduced from $n k T$ to zero if all matter is locked in the condensations. Since in the theory of general relativity, pressure contributes to the gravitational attraction, Lemaitre showed that the reduction of pressure would give rise to a reduction of the attractive forces and consequently lead to expansion of the static model leading to the Eddington-Lemaitre model. This result is not correct because it ignores the pressure due to radiation which must accompany the formation of bound condensations. The amount of energy radiated away must equal the final binding energy of the condensations. For galaxies the energy radiated per unit rest mass is approximately equal to $\left(v_{r} / c\right)^{2} \sim 10^{-5}$ where $v_{r}$ is the average rotational velocity of the Galaxy. Thus, when galaxies are formed the increase in pressure per unit rest mass is about $10^{-5}$, while the reduction in pressure due to the process suggested by Lemaitre cannot exceed $k T / m c^{2} \sim T / 10^{13} \mathrm{~K}$ for a gas consisting of protons. Thus, unless the original gas temperature exceeded $10^{8} \mathrm{~K}$ the formation of condensations increases the pressure increasing the gravitational attraction which leads to the collapse of the static model. (Note that the density of energy including the rest mass energy is unchanged during the formation of condensations.)

For Lemaitre models with long quasi-static periods, the added attraction due to radiated binding energy of the condensations slows down the rate of the expansion and it may even lead to collapse of these models. Whether or not the latter possibility will take place depends on the length of the quasi-static period and the rate of the formation and the binding energy of the condensations. Brecher and Silk (1969) have shown that if most galaxies were formed during the quasi-static period and if this period is very long, $\varepsilon<10^{-8}$, then these models also collapse back into a singularity. It can be concluded then that although formation of galaxies is easier in Lemaitre models as compared to the conventional models, the formation of galaxies from statistical fluctuations is unlikely.

\subsection{THE REDSHIFT-MAGNITUDE RELATION}

For small redshifts the magnitude-redshift relation can be approximated as

$$
m=\text { const }+5 \log z-1.08\left(q_{0}-1\right) z .
$$

After the quasi-static period Lemaitre models expand with acceleration and when 
the density of matter $\varrho_{0} \ll \varrho_{v}$ the deceleration parameter is close to $q_{0} \approx-1$. The observed redshift-visual magnitude relation for bright members of clusters of galaxies indicates models with deceleration parameters $q_{0} \approx 0.03 \pm 0.4$ (Peach, 1972). This would be consistent with Lemaitre models if the difference $\Delta m / \Delta z \approx-1.1 \mathrm{mag}$. (between $q_{0} \approx-1$ models and observed $q_{0} \approx 0$ ) can be attributed to evolution of the galaxies. With $H_{0}=50 \mathrm{~km} \mathrm{~s}^{-1} \mathrm{Mpc}^{-1}$ this requires dimming of the galaxies by 0.05 mag. $\mathrm{yr}^{-9}$. Evidently such an evolution is possible for the galaxies under consideration (Tinsley, 1972).

\subsection{RADIO SOURCE COUNTS}

Since the reintroduction of the Lemaître models by Petrosian et al. (1967), various investigators have examined the radio source counts in these models (Kardashev, 1968; McVittie and Stabell, 1968; Rowan-Robinson, 1968; Petrosian, 1969; Longair and Scheuer, 1970). In the 1969 paper I analyzed the cumulative source counts and showed that although for the Lemaitre models the slope of the $\log N \log S$ relation can be steeper than that in conventional models, the slope cannot exceed the value -1.5 expected from the Euclidean (flat, static) model. It was found that mild evolution (co-moving density of sources increasing as $(1+z)^{1.5}$ ) was necessary to obtain the observed $\log N \log S$ relation of the Cambridge catalogues. Similar results were obtained by Longair and Scheuer (1970).

In view of recent interest in differential counts, I will here compare the observed differential counts with the predictions of Lemaitre models. The results are summarized on Figure 2 where I present the variation with flux density of the ratio of the differential count $n(S)$ to the count $n_{0} \propto S^{-2.5}$ expected from the Euclidean model. The observed points are taken from the review paper by Kellermann (1972). The thin solid line is the differential count expected from uniformly distributed sources with radio luminosity at $408 \mathrm{MHz}$ of $F(408) \approx 10^{29.4} \mathrm{~W} \mathrm{~Hz}^{-1}$ in a Lemaître model with $\lambda=1.02$ and $z_{s}=2$. This curve reproduces the observed feature at high flux densities. However, when the contribution from sources with different radio luminosities are added, the narrow feature extending one decade (from 5 to 50 flux units) is washed out by the large dispersion in the radio luminosity of the sources. This is shown by the heavy solid line which has been obtained by integrating the distribution shown by the thin line over all luminosities assuming a radio luminosity function $\Psi(F) \propto F^{-2}$ for $10^{25} \mathrm{~W} \mathrm{~Hz}^{-1}<F<10^{29} \mathrm{~W} \mathrm{~Hz}^{-1}$, and $\Psi(F)=0$, otherwise. As is evident from Figure 2 this model predicts twice as many sources as observed at flux densities where the cumulative count of sources is about $10 \mathrm{sr}^{-1}$ (see the top coordinate). This discrepancy amounts to the absence of 5 sources $\mathrm{sr}^{-1}$ (or in total 60 sources) with flux densities $S_{408}>10$ flux units. In fact, for this model to agree with observation it is only necessary to assume the absence of sources with low luminosities in a local region extending to a redshift of less than unity. This is equivalent to a radio luminosity function which was steeper in the past. Or alternatively, as suggested before, mild density evolution, $\Psi(F, z) \propto(1+z)^{3 / 2}$, for redshifts less than the redshift of the quasi-static period can account for the discrepancy. The need for such evolution 


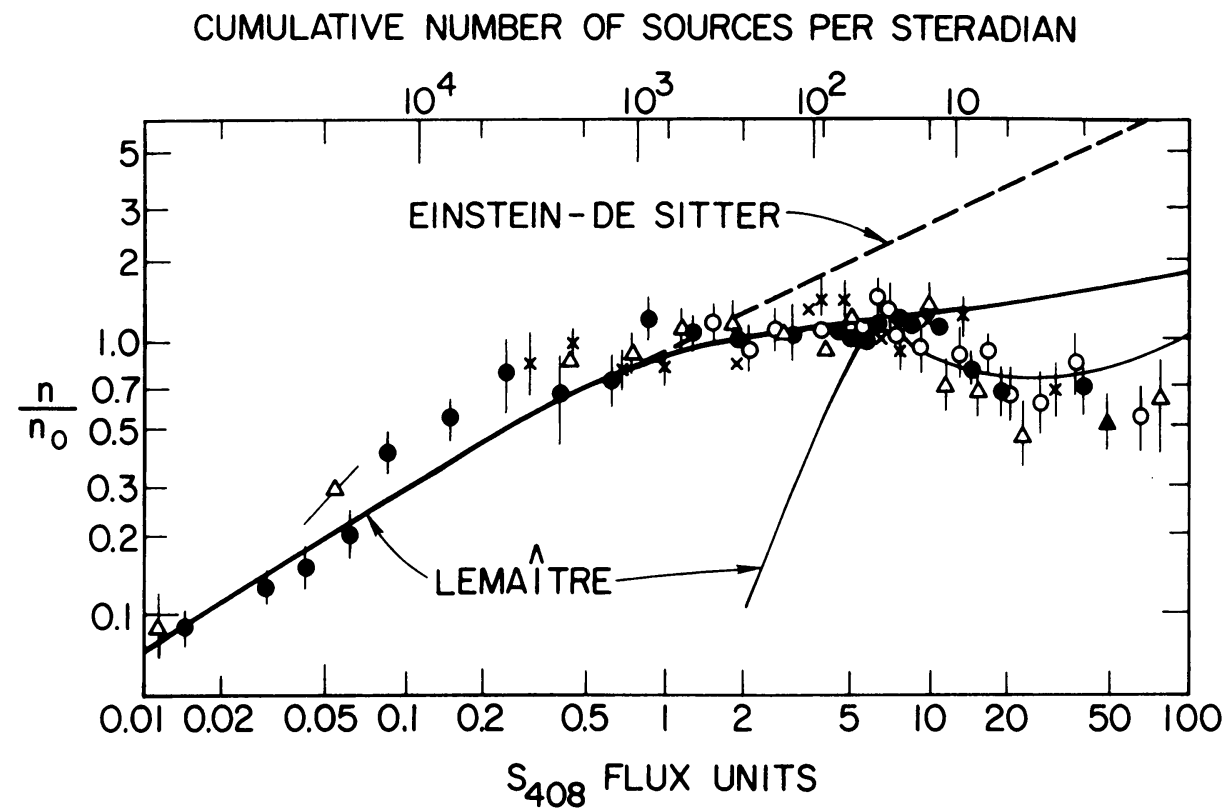

Fig. 2. The differential count of radio sources in units of the counts $\left(n_{0} \propto S^{-2.5}\right)$ expected in Euclidean universe. The points are taken from Kellermann (1972); at $75 \mathrm{~cm}, O$ at $20 \mathrm{~cm}, \triangle$ at $11 \mathrm{~cm}$ and $\times$ at $6 \mathrm{~cm}$. The solid lines are for the Lemaitre model with $\chi=1.02$ and $z_{s}=2$; the thin line is for standard candles with luminosity $10^{29.4} \mathrm{w} \mathrm{Hz}^{-1} \times\left(50 \mathrm{~km} \mathrm{~s}^{-1} \mathrm{Mpc}^{-1} / H_{0}\right)$ at $408 \mathrm{MHz}$, and the solid line for the radio luminosity function described in the text. The dashed line is the expected count in the model with $\Lambda=0$ and $q_{0}=\frac{1}{2}$ with a similar luminosity function.

detracts from the Lemaitre models but cannot be taken as evidence against them. As is well known, the discrepancy between the conventional cosmological models without evolution and observation is much greater. The dashed line in Figure 2, which shows the expected differential count in the Einstein-de Sitter model $(\Lambda=0$, $\sigma_{0}=\frac{1}{2}$ ) with a similar luminosity function, begins to deviate from observation at flux densities where the cumulative count is about $100 \mathrm{sr}^{-1}$. There are ten times more sources missing in this model as compared with the Lemaitre model.

However, for the Lemaitre model to be correct, it should also agree with the redshift distribution of the sources. According to this model, most of the sources with flux densities $S_{408}<2 \times 10^{-26} \mathrm{~W} \mathrm{~m}^{-2} \mathrm{~Hz}^{-1}$ should have redshift greater than 1.5 and a fair fraction should have redshift about 2 . There do not exist sufficient data to determine whether this is true or not. For example, of all quasi-stellar radio sources in the catalogue of Deveny et al. (1971) with $S_{408}<5 \times 10^{-26} \mathrm{~W} \mathrm{~m}^{-2} \mathrm{~Hz}^{-1}, 75 \%$ have redshifts greater than 1.4 with about $30 \%$ having redshifts $2.1 \pm 0.2$. Since this sample is not a complete sample and since radio galaxies are not included in it, no firm conclusions can be reached from it.

\subsection{REDShIFT DISTRIBUTION OF QUASARS}

Lemaitre models were re-introduced in 1967 (Petrosian et al., 1967) when the preponderance of quasars with redshifts near 2 was reported (Burbidge and Burbidge, 
1967). At that time two anomalies seemed to be present in the redshift distribution of quasars. One was the presence of a narrow spike in the redshift (both emission and absorption) distribution of the quasars near $z=1.95$. Shklovsky (1967) and Kardashev (1968) attempted to explain this feature as absorption of quasar light by intervening galaxies during the quasi-static period. A long quasi-static period was necessary to explain the narrow feature. Subsequent observations have failed to support the reality of the $z=1.95$ feature. There are now few sources with redshift larger than 2 without an absorption redshift near 1.95. We shall not discuss this effect here.

The other anomaly was the presence of a mild hump in the redshift distribution of quasars at $z \sim 2$ (with a rapid cutoff beyond it) which prompted us to consider a Lemaitre model with fairly short quasi-static period. The present observational situation in this case is the same as at the time of the previous review of this subject (Petrosian and Salpeter, 1970), except that now there are sources with redshifts larger than $z_{s} \sim 2$. As we shall see below this is strong evidence against the Lemaitre models.

Instead of comparing the number distribution of the redshifts of quasars, we compare, as before, the sum of intensities $\sum I$ of sources at a given redshift vs redshift. The reason for this is twofold: first, both the intensity and number of sources at redshifts around the antipodes $(u \approx n \pi)$ behave anomalously. For homogeneous models the intensity diverges and the differential (with respect to $u$ ) number of sources tends to zero at $u \rightarrow n \pi$. The inhomogeneities, due to their gravitational lens effect, modify the intensity of the sources and give rise to multiple (but weaker) images of a source near the antipode. The extent of both of these effects depends on the uncertain parameters which characterize the inhomogeneities. However, the total intensity due to multiple images is equal to the intensity of the sources as if the inhomogeneities were absent. Thus, the expected integrated $\sum I$ is independent of the degree of inhomogeneity and is a well behaved function of redshift; the function $F_{\alpha}(z)$ is given by

$$
F_{\alpha}(z)=\frac{1}{(1+z)^{1+\alpha}} \frac{\mathrm{d} u}{\mathrm{~d} z} .
$$

The second reason for considering $\sum I$ instead of number of sources has to do with the selection effects. The observed distributions are affected by selection effects. For the variation of the number of sources with redshift, the selection effects depend strongly on the luminosity function (since this function in general decreases rapidly with luminosity) and become uncertain when the sample is not complete to a limiting observed magnitude or flux density. On the other hand the distribution of $\sum I$ depends more weakly on the luminosity function and the lack of completeness of the sample does not effect the brighter objects which are the major contributors to $\sum I$. Furthermore, we will be interested in the distribution near $z=z_{s}$ where because of the brightening of the objects the selection effects become negligible, while it is exactly here that the distribution of the numbers behaves anomalously.

Figures 3 and 4 summarize the results. In Figure 3 we compare the observed histogram of $\sum I$ (obtained from the list of Deveny et al., 1971) with the prediction of 


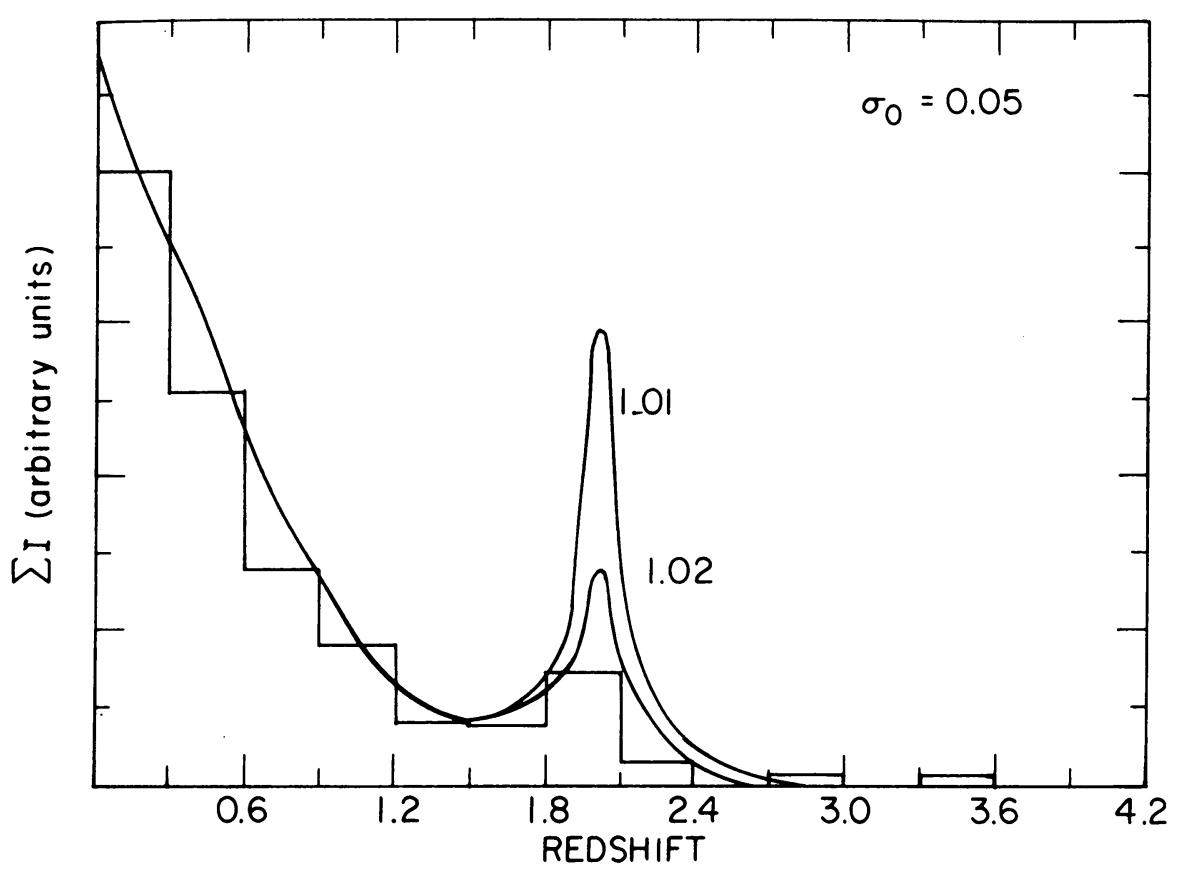

Fig. 3. Comparison of the integrated optical intensity of sources in the Deveny et al. (1971) catalog (the histogram) with the prediction of the Lemaitre models with $z_{s}=2$. The lines represent $F_{\alpha}(z) \phi(z)$ discussed in the text. The numbers on the curves give the value of $\lambda=\Lambda / \Lambda_{c}$.

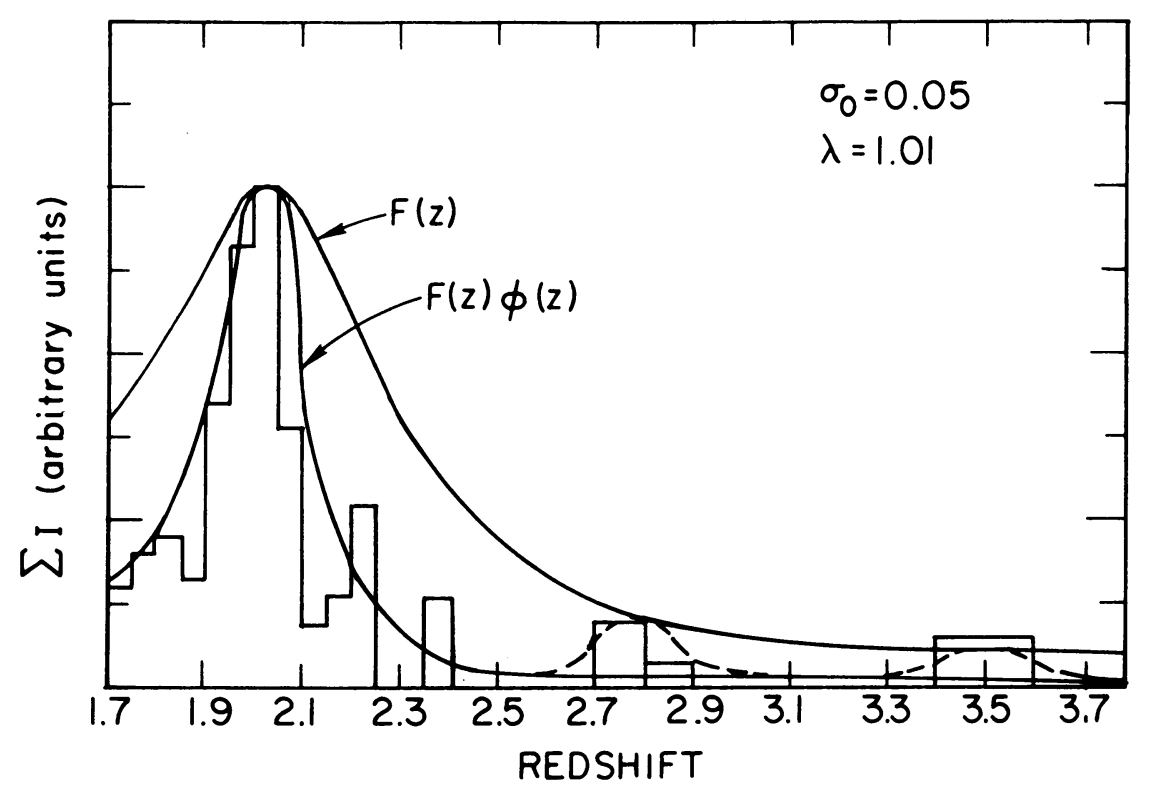

Fig. 4. Expanded version of Figure 3 near and beyond the redshift of the quasistatic period. The top solid line neglects selection effects. The lower solid lines include the selection effects as described in the text. The dashed lines around $z=2.8$ and 3.5 would result if these redshifts correspond to an antipode $u=i \pi$, $i=2,3, \ldots, n$. As explained in the text, the presence of only one such antipode is possible beyond the redshift of the quasistatic period. 
Lemaitre models with quasi-static period redshift $z_{s}=2$. What is plotted here is the function $F_{\alpha}(z) \phi(z)$ [for $\left.\alpha=0.7\right]$, where $\phi(z)$ accounts for the selection effects, and depends on the luminosity function and the assumed limiting magnitude of the sample. These effects are most important around redshift 0.5 to 1.5. Since the sample does not have any well defined radio or optical limit, the exact behavior of $\phi(z)$ is not well known. We find, however, if we assume the sample to be complete to a $408 \mathrm{MHz}$ flux density of $5 \times 10^{-26} \mathrm{~W} \mathrm{~m}^{-2} \mathrm{~Hz}^{-1}$ and assume the radio luminosity function $\Psi(F) \propto F^{-2}$ then we find it necessary to invoke mild evolution (as in the radio source counts) for redshifts less than 1.5 in order to obtain a fit at these redshifts.

Figure 4 shows an expanded version of Figure 3 for redshifts near and beyond the quasi-static period. The histogram shows the pronounced brightening of the sources near $z=2$ which is expected from Lemaitre models and which was, and still is, the single most important observation in favor of considering these models seriously. However, as is evident from Figure 4, the Lemaitre models predict very few sources for redshifts larger than 2.5 especially if the selection effects are included. Recent observations have discovered four sources with large redshifts, two of them around $z \sim 2.8$ and two near $z \sim 3.5$. Note that the histogram at these redshifts agrees with the $F_{\alpha}(z)$ curve, i.e., with the expected distribution when selection effects are negligible. Since the selection effects become negligible near an antipode the existence of one of these two humps (if they are in fact isolated humps) could be explained by assuming that the redshift of the last ghost images $\left(z_{g, n}\right)$ falls near the center of the hump. The dashed lines show the expected distribution in case of such an eventuality. However, as mentioned in connection with Equation (16), there could exist only one ghost image with a redshift significantly different from $z_{s}$. For example, the model with $\lambda=1.0098$ (cf. Equation (16)) has its second (its last) ghost image at $z \approx 3.5$. This however leaves the hump at redshift $z \sim 2.8$ unexplained. This, therefore, must be considered strong evidence against the Lemaitre models unless, because of the tendency nowadays of publishing only sources with larger redshifts, these humps are not as significant as they appear on Figure 4.

\section{Summary and Conclusions}

As mentioned in the introduction the cosmological constant was introduced on three occasions to explain some cosmological observations. It was first introduced by Einstein to obtain a static universe as was then thought to be the state of our Universe. The subsequent discovery of the expansion of the Universe removed this original motivation for its introduction. In the period following the discovery of the expansion of the Universe, Eddington and Lemaître argued in favor of the retention of the cosmological constant while Einstein and others favored its abandonment. Lemaître, in particular, favored cosmological models with long quasi-static periods (which have come to be known as Lemaitre models) because they could explain the fact that the Hubble age $\left(H_{0}^{-1}\right)$ was smaller than the age of the solar system and because 
they facilitated the formation of galaxies. Subsequent observations have steadily increased the Hubble age and have again removed this second motivation for considering models with a non-zero cosmological constant.

Finally after the discovery of quasars we (with Salpeter and Szekeres) re-introduced the cosmological constant and the Lemaître models in order to explain the preponderance of quasars near redshift 2, without having to invoke arbitrary evolutions which are required in conventional models. As shown in Sections 4.3 and 4.4, the accumulated observational data since 1968 speak against the Lemaître models without evolution. In particular the existence of quasars with redshifts larger than 2.5 provide strong evidence for abandoning the Lemaitre models once again. Since the Lemaître models, because of their drastically different observational character from the rest of general relativistic models, provide the only cosmological motivation for retention of the cosmological constant this also means abandoning the cosmological constant.

These, however, by no means are evidence either against the Lemaitre models or the cosmological constant. Observations only set the limit

$$
|\Lambda|<2 \times 10^{-56} \mathrm{~cm}^{-2}
$$

on the cosmological constant. Furthermore, Lemaitre models with evolution are still a possibility. We have demanded more from Lemaitre models by requiring them to explain observations without evolution while we accept conventional models which would need very complicated evolution to explain the pronounced hump near $z=2$ in Figure 4 if it is real.

But in the absence of strong evidence in favor of Lemaitre models we must once again send back the Lemaitre models and along with it the cosmological constant to the shelf until their next re-appearance.

\section{Acknowledgements}

This work was supported in part by the National Aeronautics and Space Administration under Grant NGR 05-020-510.

\section{References}

Bonnor, W. B.: 1957, Monthly Notices Roy. Astron. Soc. 117, 104.

Brecher, K. and Silk, J.: 1969, Astrophys. J. 158, 91.

Burbidge, G. R. and Burbidge, E. M.: 1967, Astrophys. J. Letters 148, L107.

DeVeny, J. B., Osborn, W. H., and Janes, K.: 1971, Publ. Astron. Soc. Pacific 83, 611.

Eddington, A. S. : 1939, Sci. Prog. 34, 225.

Ginzburg, V. L.: 1971, Comments Astrophys. Space Phys. 3, 7.

Iben, I. and Faulkner, J.: 1968, Astrophys. J. 153, 101.

Kardashev, N.: 1967, Astrophys. J. Letters 150, L135.

Kellermann, K. I.: 1972, Astron. J. 77, 531.

Lemaître, G. : 1931, Monthly Notices Roy. Astron. Soc. 90, 490.

Longair, M. S. and Scheuer, P. A. G.: 1970, Monthly Notices Roy. Astron. Soc. 151, 45.

McVittie, G. C. and Stabell, R. : 1968, Astrophys. J. Letters 150, L141. 
North, J. D.: 1965, The Measure of the Universe, Clarendon Press, Oxford, pp. 83-92 and p. 125.

Peach, J. V.: 1972, in D. S. Evans (ed.), 'External Galaxies and Quasi-Stellar Objects', IAU Symp. $44,314$.

Petrosian, V.: 1969, Astrophys. J. 155, 1029.

Petrosian, V. and Ekers, R. D.: 1969, Nature 224, 484.

Petrosian, V. and Salpeter, E. E.: 1968, Astrophys. J. 151, 411.

Petrosian, V. and Salpeter, E. E.: 1970, Comments Astrophys. Space Sci. 2, 109.

Petrosian, V., Salpeter, E. E., and Szekeres, P.: 1967, Astrophys. J. 147, 1222.

Rindler, W.: 1969, Essential Relativity, Van Nostrand Reinhold, New York, pp. 80, 81.

Rowan-Robinson, M.: 1968, Monthly Notices Roy. Astron. Soc. 141, 445.

Sandage, A.: 1972a, Astrophys. J. 178, 1.

Sandage, A.: 1972b, Astrophys. J. 178, 485.

Shklovsky, I.: 1967, Astrophys. J. Letters 150, L1.

Solheim, J. E.: 1968, Nature 219, 415.

Tinsley, B. M.: 1972, Astrophys. J. Letters 173, L93.

Zel'dovich, Ya. B. : 1967, JETP Letters 6, 1345.

Zel'dovich, Ya. B.: 1968, Soviet Phys. Uspekhi 11, 381. 